\title{
INNOVACIÓN EDUCATIVA DESDE LA ÓPTICA DE LOS DOCENTES EN UNA IES. UN ESTUDIO COMPARATIVO ${ }^{123}$
}

Educational innovation from the teachers' point of view in a SEI. A comparative study

1- Autor: Susana Céspedes Gallegos. Grado académico: Maestría en Ciencias Administrativas. Adscripción: Tecnológico Nacional de México Campus Coatzacoalcos. Correo electrónico: scespedesg@itesco.edu.mx. ORCID ID: https://orcid. org/0000-0001-5035-207X

2- Co-Autor 1: Luis Armando Vázquez González. Grado académico: Estudiante de Maestría en Ciencias Administrativas. Adscripción: Tecnológico Nacional de México Campus Coatzacoalcos. Correo electrónico: lvazquezg@itesco.edu.mx. ORCID ID: https://orcid.org/0000-0001-5765-5559

3- Co-Autor 2: Maribel Domínguez Vargas*. Grado académico: Doctorado en Administración. Adscripción: Tecnológico Nacional de México Campus Costa Chica. Correo electrónico: maribel.d@costachica.tecnm.mx. ORCID ID: https://orcid. org/0000-0001-8057-9769. *autor de correspondencia 


\title{
RESUMEN:
}

Ante la denominada $4^{a}$ Revolución Industrial que hace referencia a las tecnologías digitales, a la física y a la biología, los docentes vistos como colaboradores de una IES deben contar con los elementos necesarios para enfrentar los retos emergentes que conlleva. El estudio es de tipo descriptivo, cuantitativo y de tipo transversal con un corte único en el tiempo, semestre febrero-junio del 2021. Se aplicó un instrumento con un Alfa de Cronbach de 0.887 sobre conducta intraemprendedora, el cual consta de siete dimensiones: innovación, asunción de riesgos, apoyo de la dirección, libertad en el trabajo, recompensas, tiempo disponible e incertidumbre en las tareas; consta de 58 items. Los principales hallazgos en ambas instituciones educativas sobre la percepción de innovación educativa fueron en la dimensión D4 libertad en el trabajo y su relación positiva con el género y la edad a través de la correlación de Pearson y Spearman.

Palabras clave: Innovación; Educación; Mercado Laboral, Capital Humano.

\begin{abstract}
:
Facing the so-called 4th Industrial Revolution that refers to digital technologies, physics and biology, teachers seen as collaborators of a SEI must have the necessary elements to confront the appearing emerging challenges. The study is descriptive, quantitative, and cross-sectional with a single cut in time, february-june 2021 semester. An instrument with a Cronbach's Alpha on intrapreneurial behavior was applied, consisting of seven dimensions: innovation, risk assumption, management support, freedom at work, rewards, available time, and task uncertainty; consists of 58 items. Main findings in both educational institutions on the perception of educational innovation were at dimension D4 freedom at work and its positive relationship with gender and age through the Pearson and Spearman correlation.
\end{abstract}

Keywords: Innovation; Education; Labor Demand; Human Capital.

Clasificación JEL: O31, I21, J23, J24 


\section{Introducción}

Diversos temas han cobrado especial atención ante el surgimiento de la actual pandemia COVID-19: economía creativa, gestión del conocimiento, aprendizajes emergentes, inteligencia emocional, competencias y habilidades gerenciales, entre otros; en este sentido, la innovación en las Instituciones de Educación Superior, IES, no es la excepción, ante tales retos y circunstancias las IES deben enfocar sus esfuerzos a la preparación académica, intelectual, cultural, laboral, científica de acuerdo a las necesidades inherentes que require el Mercado laboral: nuevas competencias y habilidades para la solución de problemas en un entorno competitivo así como también a las iniciativas de los propios estudiantes para emprender sus propias ideas creativas. En este sentido, los profesores son el elemento principal para transmitir conocimientos, habilidades, experiencias, emociones, aprendizajes que impliquen una transformación, un cambio en la conducta y pensamientos de la comunidad estudiantil, para actuar de manera distinta ante la solución de problemas que enfrentan en el mundo actual. Es por ello la necesidad de identificar en los profesores la perspectiva sobre la innovación educativa, para desarrollar acciones y estretagias que permitan alcanzar los objetivos institucionales de un contexto educativo y que se traduzcan en resultados evidenciables: publicación de artículos científicos en revistas indexadas y de alto impacto tanto a nivel nacional e internacional, registro de marca y patente, desarrollo de nuevos productos y servicios, vinculación con el sector empresarial, científico y servicio a la comunidad.

\section{Revisión de literatura}

\subsection{Innovación en las instituciones educativas}

La educación y la competitividad son elementos primordiales en la formación académica de los estudiantes en una IES, en este sentido, la innovación debe convertirse en un ingrediente esencial para satisfacer las necesidades del mercado laboral de acuerdo con Silva (2017). Para Jiménez (2017) un factor relevante en las IES es la adopción del cambio en la cultura institucional, se necesita que los líderes tengan una visión sobre las metas a alcanzar, se diseñen estrategias, incentivos, se fomente el trabajo colaborativo, pero, sobre todo, que los docentes sean gestores del cambio en la comunidad estudiantil como punto de partida hacia la innovación. Dentro de las IES, Ramírez y Montoya (2018) consideran que para que exista innovación, la buena docencia es la parte elemental, ya que consiste en motivar a los estudiantes, crear un ambiente de aprendizaje, establecer vínculos con la carrera, tener presente la misión y visión institucional, fomentar la evaluación docente y promocionar y difundir el aprendizaje entre la comunidad estudiantil.

Es importante reconocer que, en las IES, los docentes tienen un papel importante para fomentar la cultura de la innovación en los estudiantes, pero de acuerdo con Medina, Ruíz y Pérez (2019), son precisamente los profesores quienes no poseen las competencias de investigación e innovación, por lo tanto, es necesario considerar estrategias de actualización docente para subsanar esa debilidad en los mismos. En ese mismo sentido, Palomera, Briones, y Gómez, (2019) consideran que para que exista innovación en las IES, los docentes necesitan enriquecer sus valores y competencias personales para mejorar el trabajo colaborativo. 
Para que exista innovación en las instituciones, Fidalgo-Blanco (2020) establecen que, es importante que los docentes cuenten con los elementos necesarios: conocimientos, herramientas, tecnología y además de una metodología a seguir para encontrar la mejor forma idónea de innovar. Impulsar la capacidad reflexiva en los estudiantes, promover el pensamiento crítico, desarrollar las competencias, evaluar el aprendizaje en los estudiantes, impulsar la generación del conocimiento en las IES, elementos indispensables para lograr la innovación en la comunidad estudiantil, según Pascual, López y Hamodi (2019). Un primer paso para que exista la innovación en las IES, de acuerdo con Pesantéz (2020) es la investigación, los profesores deben saber identificar los problemas existentes en sus instituciones, para iniciar el proceso de investigación, detectar problemas y sugerir soluciones pertinentes, para ello, los docentes deben aplicar sus competencias y saberes para determinar soluciones idóneas, creativas e innovadoras.

De forma similar, Ríos y Ruíz (2020) consideran que en América Latina, la innovación educativa, consiste en tener una visión sistémica, una valorización del trabajo docente, contar con recursos y herramientas tecnológicas, medios de comunicación eficientes, que permitan transformar los factores que intervienen en el aprendizaje: programas de estudio, estrategias didácticas, cultura institucional , responsabilidad de los estudiantes, formas de evaluación, entre otros, así como también el trabajo de las autoridades educativas. Todos estos elementos son acciones conjuntas para lograr una educación de calidad, incluyente y diversa, pero también justa y equitativa que permita favorecer la innovación en el contexto educativo.

\subsection{Potencialización de la innovación en un contexto educativo}

En este mismo orden de ideas, Sancho (2018) apuesta que la innovación en las IES es un desafío y no debe observarse como una moda, sino al contrario, que sirva para analizar el trabajo que realizan los docentes, cuestionarse cómo aprenden los estudiantes, detectar necesidades de conocimientos, tecnologías, experiencias de aprendizajes y los sistemas de evaluación de tal forma que permita tener una perspectiva del trabajo de las instituciones.

Según Barraza (citado por Zavala-Guirado, González-Castro y Vázquez-García, 2020), consideran que la innovación en un contexto educativo consiste en una serie de transformaciones y cambios en el ejercicio de la docencia, enriquecer con nuevos temas los programas de estudio, utilizar nuevos recursos apoyados en la tecnología, el uso de una nueva pedagogía en el proceso de enseñanza, pero sobre todo un cambio de actitud. Para Leal-Fonseca, Rojas, Ortiz-Pradilla y Monroy-Osorio (2020), la innovación es un proceso sistémico en las IES, porque promueve la solución de problemas con la finalidad de establecer mejoras en el sistema.

Desde otro ángulo de análisis, la innovación educativa para Oseda, Mendivel y Durán (2020) en un conjunto de factores ideológicos, sociales y económicos, para cual se requiere que las IES se vinculen con la sociedad, el sector productivo y algunas instancias que favorezcan la producción y el trabajo colaborativo. Klingner y Boardman (citados por Ramírez y Lugo-Ocando, 2020) señalan que la tecnología, la investigación, los procesos y establecer nuevos cambios en el trabajo y la educación, se obtiene como resultado la innovación. En la nueva forma de transmitir conocimiento, se ha tenido la necesidad de utilizar la realidad virtual, de acuerdo con lo planteado por la Organización de las Naciones Unidas para 
la Educación, la Ciencia y la Cultura, UNESCO (citado por Botella, Hurtado y Ramo, 2018), es que en los tiempos actuales se requiere de nuevos cambios que se adapten a la nueva normalidad de enseñanza

Una de las declaraciones de la Organización de las Naciones Unidas, ONU, sobre innovación educativa, es que constituye un cambio en la educación, para mejorar la calidad de las actividades que se desarrollan en el ámbito educacional; sin embargo, para lograrlo se necesitan de todos los actores, de la realización de prácticas y aplicaciones que provoquen una nueva cultura de acuerdo con las afirmaciones de Zavala-Guirado et al. (2020). En este sentido Prendes y Cerdán (2021) consideran que las tecnologías digitales han tenido una gran aplicación en diferentes áreas, sin excepción en el campo de la educación, que sin duda ha sido unos de los recursos más utilizados en el período de la pandemia COVID-19. La tecnología evoluciona constantemente de tal forma que obliga a las IES a aprender de manera más rápida para permanecer actualizados.

En su contraparte, Zulueta (2020) establece que en las IES existen normas y reglamentos que regulan la puntual vigilancia de las actividades docentes, sin embargo, la inspección educativa siempre ha sido molesta para los docentes porque consideran que es una falta a la libertad de enseñanza y en algunos casos lo tipifican con una persecución laboral. Sein-Echaluce, Fidalgo-Blanco y García-Peñalvo (2019) consideran que no existe un modelo de innovación educativa que sea único en las IES, cada institución es diferente en sus procesos de enseñanza-aprendizaje y por ende los resultados son diferentes, existen elementos claves como, por ejemplo, la instrucción de los profesores, la pertinencia de la materia, los métodos y técnicas utilizados.

Para la presente investigación toma como referencia el modelo de la conducta intraemprendedora, el cual establece que para que exista innovación en las organizaciones e instituciones es importante que exista el desarrollo de la creatividad entre sus miembros, para dar lugar al surgimiento de nuevas ideas, procesos, productos, servicios y formas de trabajo, por ende, la creatividad es el primer paso a la innovación.

De acuerdo con Hornsby, Kuratko y Zahra (citados por Moriano, Topa, Valero y Lévy, 2009) establecen los factores que integran el modelo de la conducta intraemprendedora, entre los que destacan:

- Innovación: implicación de un cambio de mentalidad y de iniciativa para fomentar la cultura de la innovación.

- Asunción de riesgos: conceptualización del fracaso y la implicación del riesgo en la generación de ideas creativas.

- Apoyo de la dirección: es indispensable que los directivos promuevan el apoyo de la conducta intraemprendedora de los colaboradores y a su vez proporcionen los elementos necesarios para su realización.

- Libertad en el trabajo: brindar la oportunidad del trabajo autónomo entre los colaboradores, la libertad en la toma de decisiones y permitir los errores como parte del proceso de innovar.

- Recompensas: enfocadas en los logros y resultados obtenidos para motivar el trabajo realizado entre los colaboradores.

- Tiempo disponible: los colaboradores requieren de un período para la generación de ideas creativas para someterlas a prueba y error.

- Incertidumbre en las tareas: la productividad de los colaboradores en relación con los resultados esperados y los indicadores alcanzados. 
La innovación educativa depende en primera instancia de la conducta intraemprendedora de sus colaboradores y del aprovechamiento de las ideas de estos, en este sentido las ideas deben ser congruentes, relevantes y trascendentes. En este ejercicio educativo, los profesores, son el primer eslabón en la institución educativa para contribuir a través del desarrollo de sus talentos y habilidades con la innovación y transmitirla a sus estudiantes, para tal efecto Clara y Vega (2021) consideran que una educación de calidad radica en sus procesos de enseñanza-aprendizaje, por lo que la calidad se define en términos de excelencia, perfección, valor económico y transformación. En este ámbito, la innovación es un primer eslabón hacia la calidad educativa. Una vez identificada la teoría y las variables de estudio se plantean a continuación las siguientes preguntas de investigación: 1. ¿Cuáles son las dimensiones de innovación educativa que perciben los docentes de ambas instituciones educativas? y 2. ¿Cuál es la dimensión que más relación tiene para innovar en un contexto educativo tanto del Tecnm Campus Coatzacoalcos y Costa Chica?

\section{Metodología}

El presente estudio que aquí se analiza, muestra una metodología cuantitativa, de alcance descriptivo y de tipo transversal con un corte único en el tiempo, ya que los estudios se realizaron en el semestre febrero-junio del presente año con la finalidad de identificar los determinantes de la innovación en un contexto educativo. Se determinó una muestra de tipo no probabilístico por conveniencia, derivado de la pertinencia y proximidad de los investigadores según Ávila (2006) de 12 docentes del Tecnm Campus Costa Chica y 58 docentes del Tecnm Campus Coatzacoalcos, se aplicó un instrumento de medición de Moriano et al. (2009) sobre conducta intraemprendedora, con un Alfa de Cronbach de 0.887, lo cual establece su confiablidad de acuerdo con Hernández, Fernández y Baptista (2014), presenta una escala de Likert, donde 1 es totalmente en desacuerdo y 5 totalmente de acuerdo. El instrumento se encuentra estructurado en 7 dimensiones, entre los que destacan: innovación, asunción de riesgos, apoyo de la dirección, libertad en el trabajo, recompensas, tiempo disponible e incertidumbre en las tareas y 58 ítems. El cuestionario fue aplicado a través de Google form a los docentes de ambas instituciones educativas, derivado de la persistencia de la actual pandemia COVID-19.

\section{Objetivo General}

Analizar los determinantes de la conducta intraemprendedora de los docentes y en un contexto educativo.

\section{Hipótesis de investigación}

Derivado de la revisión de la literatura y de la construcción del marco teórico se propone la siguiente hipótesis de investigación Hi: la dimensión que más se relaciona con la innovación educativa en las IES, es la dimensión D2 asunción de riesgos.

\section{Análisis de datos}

Una vez realizado el diseño metodológico se procedió a la captura de la información en el Statistical Package for the Social Science, SPSS, cuyos resultados se analizan a continuación. En la Tabla 1 se analizan los datos sociodemográficos de los profesores del Tecnm Campus Coatzacoalcos 
DOI: http://dx.doi.org/10.20983/novarua.2021.23.5

Tabla 1. Datos sociodemográficos de los profesores del Tecnm Campus Coatzacoalcos

\begin{tabular}{|c|c|c|c|c|}
\hline Carrera & Edad & Género & Tipo de plaza & Antigüedad \\
\hline $\begin{array}{l}\text { Ing. Industrial } \\
30 \%\end{array}$ & $22-32: \quad 5 \%$ & Masculino: $54.2 \%$ & PTC: $40.7 \%$ & $\begin{array}{c}\text { 01-10 Años: } \\
30 \%\end{array}$ \\
\hline $\begin{array}{l}\text { Ing. Administra- } \\
\text { ción } 35 \%\end{array}$ & $33-43: 30.6 \%$ & Femenino: $45.8 \%$ & PA: $59.3 \%$ & $\begin{array}{c}\text { 11-20 Años: } \\
59 \%\end{array}$ \\
\hline \multirow[t]{2}{*}{$\begin{array}{c}\text { Ing. Gestión } \\
\text { Empresarial } \\
35 \%\end{array}$} & 44-54: $52.5 \%$ & Otro: & PT: $0 \%$ & $\begin{array}{c}\text { 21- } 22 \text { Años: } \\
11 \%\end{array}$ \\
\hline & $\begin{array}{c}\text { 55-60: } 11.9 \% \\
\text { Total: } 100 \%\end{array}$ & Total: $100 \%$ & Total: $100 \%$ & Total: $100 \%$ \\
\hline Total: $100 \%$ & & & & \\
\hline
\end{tabular}

Fuente: Elaboración propia.

De acuerdo con la recogida de los datos de los profesores del Tecnm Campus Coatzacoalcos, el 54.2\% de los encuestados corresponde al género masculino, el 52.5\% tiene una edad entre 44 y 54 años, el 59.3\% posee una plaza de asignatura y tienen una antigüedad de entre 11 y 20 años laborando en la institución, de igual forma en la Tabla 2 se observan los datos sociodemográficos de los docentes del Tecnm Campus Costa Chica.

Tabla 2. Datos sociodemográficos de los profesores del Tecnm Campus Costa Chica

\begin{tabular}{ccccc}
\hline Carrera & Edad & Género & Tipo de plaza & Antiguiedad \\
\hline $\begin{array}{c}\text { Contador Público } \\
\text { 39\% }\end{array}$ & 22-32: $8.3 \%$ & Masculino: $33.3 \%$ & PTC: $41.7 \%$ & $\begin{array}{c}\text { 01-10 Años: } \\
33.3 \%\end{array}$ \\
$\begin{array}{c}\text { Ing. Gestión Empre- } \\
\text { sarial } \\
61 \%\end{array}$ & 33-43: $25 \%$ & Femenino: $58.3 \%$ & PA: $41.7 \%$ & $\begin{array}{c}11-20 \text { Años: } \\
41.7 \%\end{array}$ \\
& 44-54: $8.3 \%$ & Otro: $8.3 \%$ & PT: $16.7 \%$ & $21-30$ Años: \\
& $55-60: 8.3 \%$ & Total: $100 \%$ & Total: $100 \%$ & Total: $100 \%$ \\
& Total: $100 \%$ & & & \\
\hline
\end{tabular}

Fuente: Elaboración propia.

Los docentes participantes de la muestra a conveniencia están representados por el 58.3\% del género femenino, correspondiente a la carrera de Ing. Gestión Empresarial, el 25\% de los encuestados tiene una edad entre 33 y 43 años, el $41.7 \%$ es profesor de tiempo completo y tienen una antigüedad laboral entre 
DOI: http://dx.doi.org/10.20983/novarua.2021.23.5

11 y 20 años. En la Tabla 3 se muestra la media por dimensión del instrumento de medición y la media general de las instituciones educativas.

Tabla 3. Media por dimensiones y media general de las IES

\begin{tabular}{|c|c|c|}
\hline $\begin{array}{l}\text { Dimensión del instrumento } \\
\text { de medición }\end{array}$ & $\begin{array}{c}\text { Media } \\
\text { Tecnm Campus Coatzacoal- } \\
\text { cos }\end{array}$ & $\begin{array}{c}\text { Media } \\
\text { Tecnm Campus Costa } \\
\text { chica }\end{array}$ \\
\hline D1 Innovación & 4.08 & 4.05 \\
\hline D2 Asunción de riesgos & 3.86 & 3.48 \\
\hline D3 Apoyo de la dirección & 2.54 & 2.35 \\
\hline D4 Libertad en el trabajo & 3.18 & 3.24 \\
\hline D5 Empleo de recompensas & 2.81 & 2.87 \\
\hline D6 Tiempo disponible & 3.22 & 3.09 \\
\hline $\begin{array}{l}\text { D7 Incertidumbre en las } \\
\text { tareas }\end{array}$ & 3.26 & 3.23 \\
\hline Media general & 3.18 & 3.03 \\
\hline
\end{tabular}

Fuente: Elaboración propia.

De acuerdo con la Tabla 3, en ambas instituciones educativas, la dimensión que más se percibe por encima de la media general es la dimensión D1, D2, D4, D6 y D7 y la que menos se percibe por debajo de la media general es la dimensión D3 y D5 apoyo de la dirección. En este sentido, los docentes perciben estar dispuestos a trabajar y fomentar un cambio de conducta hacia la innovación, pero para ello requieren del apoyo de las autoridades educativas para lograr dichos objetivos. En esta actual situación de pandemia, resulta un reto para los docentes de una IES trabajar en aspectos relacionados con la innovación, sin embargo, el contexto educativo exige del talento y habilidades de los profesores para cumplir con la tercera misión de las IES: producción y transferencia del conocimiento, actividad emprendedora y compromiso social con la comunidad. Con los resultados obtenidos se da respuesta a la primera pregunta de investigación. A continuación, en la Tabla 4 se analiza la correlación de Spearman, una prueba no paramétrica que permite predecir y relacionar las dimensiones del presente estudio para ambas instituciones educativas. 
Tabla 4. Correlación de Spearman

\begin{tabular}{lccccccc}
$\begin{array}{c}\text { Correlación de } \\
\text { Spearman }\end{array}$ & D1 & D2 & D3 & D4 & D5 & D6 & D7 \\
\hline $\begin{array}{c}\text { Tecnm Campus } \\
\text { Coatzacoalcos }\end{array}$ & 1.000 & 0.184 & 0.172 & $.389^{* *}$ & $.292^{*}$ & 0.109 & $.310^{*}$ \\
$\begin{array}{c}\text { Tecnm Campus } \\
\text { Costa Chica }\end{array}$ & 1.000 & 0.546 & -0.070 & $.627^{*}$ & 0.039 & $.595^{*}$ & 0.307 \\
\hline
\end{tabular}

Fuente: Elaboración propia.

En este sentido, de acuerdo con la correlación de Spearman, para el Tecnm Campus Coatzacoalcos, la correlación positiva es la dimensión D4 libertad en el trabajo, bajo este resultado, los docentes necesitan de la oportunidad de tomar sus propias decisiones e iniciativas para emprender nuevas ideas en su contexto laboral educativo. De igual forma, para Tecnm Campus Costa Chica, la correlación positiva es la dimensión D4, pero en la tabla se puede observar que existe una correlación negativa en la dimensión D3 apoyo de la dirección, lo que se interpreta como la falta de intervención de la dirección para apoyar el trabajo colaborativo de los docentes de la institución. En este mismo orden de ideas, en la Tabla 5 se muestra la correlación de Pearson, una prueba paramétrica para analizar la relación entre las dimensiones.

Tabla 5. Correlación de Pearson

\begin{tabular}{lccccccc}
\hline $\begin{array}{c}\text { Correlación de } \\
\text { Pearson }\end{array}$ & D1 & D2 & D3 & D4 & D5 & D6 & D7 \\
\hline $\begin{array}{c}\text { Tecnm Campus } \\
\text { Coatzacoalcos }\end{array}$ & 1 & $.321^{*}$ & .106 & $.362^{* *}$ & $.412^{* *}$ & .089 & $.457^{* * *}$ \\
$\begin{array}{c}\text { Tecnm Campus } \\
\text { Costa Chica }\end{array}$ & 1 & $.708^{*}$ & 0.025 & $.747^{* *}$ & 0.423 & 0.416 & 0.317 \\
\hline
\end{tabular}

Fuente: Elaboración propia.

De acuerdo con los resultados obtenidos en la correlación de Pearson para el Tecnm Campus Coatzacoalcos, la dimensión que tiene una relación directa (positiva) con la innovación educativa son las dimensiones D4 libertad en el trabajo y D5 empleo de recompensas, y para el Tecnm Campus Costa Chica es la D4 libertad en el trabajo. Con los datos analizados, se responde a la segunda pregunta de investigación y, por lo tanto, la hipótesis de investigación Hi se rechaza ya que a través de la correlación de Spearman y Pearson se ha demostrado que la dimensión que más se relaciona con la innovación educativa es la dimensión D4 libertad en el trabajo y no la dimensión D2 asunción de riesgos. En la Tabla 6 se muestra la relación de la dimensión D4 libertad en el trabajo con el género y la edad a través de Chi-cuadrado. 
Tabla 6. Relación Chi-cuadrado con la dimensión D4 libertad en el trabajo con el género

\begin{tabular}{|c|c|c|c|}
\hline \multicolumn{4}{|c|}{ Pruebas de chi-cuadrado } \\
\hline & Valor & df & $\begin{array}{c}\text { Significación asintótica } \\
\text { (bilateral) }\end{array}$ \\
\hline $\begin{array}{c}\text { Chi-cuadrado de Pearson } \\
\text { Tecnm Campus Coatzacoal- } \\
\text { cos }\end{array}$ & $17.320^{\mathrm{a}}$ & 8 & 0.027 \\
\hline $\begin{array}{c}\text { Chi-cuadrado de Pearson } \\
\text { Tecnm Campus Costa Chica }\end{array}$ & $20.229^{\mathrm{a}}$ & 8 & 0.010 \\
\hline
\end{tabular}

Fuente: Elaboración propia.

La prueba de Chi-cuadrado muestra una relación positiva de la dimensión D4 libertad en trabajo con el género de los profesores de ambas instituciones. Es decir, tanto los docentes del género masculino y femenino pueden ser innovadores en una institución educativa. En este sentido, en la Tabla 7 se muestra la prueba de Chi-cuadrado en relación con la edad.

Tabla 7. Relación Chi-cuadrado con la dimensión D4 con la edad

\begin{tabular}{|c|c|c|c|}
\hline \multicolumn{4}{|c|}{ Pruebas de chi-cuadrado } \\
\hline & Valor & df & $\begin{array}{c}\text { Significación asintó- } \\
\text { tica (bilateral) }\end{array}$ \\
\hline $\begin{array}{c}\text { Chi-cuadrado de Pearson } \\
\text { Tecnm Campus Coatzacoalcos }\end{array}$ & $41.950 \mathrm{a}$ & 56 & 0.918 \\
\hline $\begin{array}{c}\text { Chi-cuadrado de Pearson } \\
\text { Tecnm Campus Costa Chica }\end{array}$ & $19.619 \mathrm{a}$ & 12 & 0.075 \\
\hline
\end{tabular}

Fuente: Elaboración propia.

De igual forma, la prueba de Chi-cuadrado muestra una relación positiva de la dimensión D4 libertad en el trabajo con la edad de los profesores de ambas instituciones institución. Lo que significa que la variable edad no es un impedimento para innovar en un contexto educativo.

\section{Conclusiones}

La innovación es un proceso que incluye elementos que intervienen directamente en la buena voluntad de los miembros que la integran, como la imaginación, la intuición, la corazonada, la creatividad y el emprendimiento, entendido como la puesta en práctica de las ideas creativas para la solución de un problema, bien para la creación de una empresa o hacia el interior de las organizaciones. Ante la situación de pandemia en un contexto educativo, se requiere que los profesores estén actualizados y capacitados para desarrollar nuevas competencias y habilidades que impacten en la formación profesional de los estudiantes. En los resultados obtenidos se puede apreciar en igualdad de condiciones sobre la percepción que observan 
los profesores del Campus Coatzacoalcos y Tecnm Campus Costa Chica con respecto a la innovación educativa, los que ellos demandan es libertad en el trabajo para realizar actividades, tomar decisiones y todo aquello que implica el quehacer educativo en sus respectivas instituciones.

Los cambios emergentes, la tecnología evolutiva, la educación en línea, el fenómeno de la globalización, los cambios económicos influyen en las IES, es por ello la importancia de reconocer que se requiere de flexibilidad para que los docentes puedan sentirse en un ambiente de aprendizaje, de tener confianza en la generación de ideas para innovar, de la comprensión de los beneficios del fracaso como parte del proceso para realizar cambios, modificaciones, transformaciones y sentirse apoyados por los directivos de sus instituciones.

Libertad en el trabajo significa que los profesores se sientan en completa libertad de actuación de cátedra, en la realización de proyectos, en el trabajo colaborativo, ya sea institucional o en un trabajo en red con otras instituciones, que les permitan poner en práctica sus ideas creativas y que sean los mismos profesores que observen sus éxitos y fracasos ante la libertad de trabajar. No debe confundirse en el término de libertad con el hecho de que los profesores puedan violentar sus derechos y obligaciones laborales institucionales. La confianza en los profesores puede marcar la pauta del camino hacia la libertad.

Las reglas y políticas rígidas que norman la conducta y el trabajo de los profesores inútilmente contribuyen a un ambiente de trabajo colaborativo e innovador. De acuerdo con la construcción del marco teórico y de la revisión de la literatura, la dimensión D3 apoyo de la dirección, es uno de factores elementales para que exista la innovación en las organizaciones e instituciones así como también la asunción de riesgos, puesto que toda innovación es susceptible de prueba y error y ende de fracasos, mismo que sirven como fundamento para enriquecer el trabajo hasta encontrar la forma idónea para llevarlo a cabo, el empleo de recompensas es un elemento que motiva a los colaboradores y equipos de trabajo, es una especie de recompensa al esfuerzo y trabajo realizado, porque la innovación implica inversión de tiempo.

El factor de tiempo libre suma a los esfuerzos de la innovación porque los docentes necesitan de un espacio y no saturación de cargas de trabajo, por ello el equilibrio en sus actividades labores puede contribuir al objetivo de los docentes innovadores. El desconocimiento e incertidumbre de las tareas del docente puede convertirse en un impedimento para la innovación, el no saber cómo, cuándo o qué hacer o seguir determinados procedimientos entorpece el trabajo colaborativo. Para que exista la innovación en las instituciones y organizaciones se debe brindar el apoyo a las ideas de los colaboradores, ya que son la primera instancia para iniciar el camino hacia la innovación.

Una de las limitantes en la investigación que aquí se sintetiza, es la poca colaboración de los profesores para la aplicación de la encuesta en ambas instituciones. Este estudio queda abierto para futuras líneas de investigación que permitan analizar y profundizar el tema de innovación educativa con otras instituciones tanto públicas como privadas.

\section{Referencias}

Ávila, B. H. (2006). Introducción a la metodología de la investigación. Eumet.Net.

Botella, N. A., Hurtado, S. A. y Ramo, S. A. (2018). Innovación educativaa traves de la realidad virtual y el paisaje sonoro. Creativity and educational innovation, (2), 113-127. DOI: https://doi.org/10.7203/CREATIVITY.1.13628. 
Clara, Z. M. y Vega, Z. C. (2021). El carácter polisémico deeducación de calidad en el nivel universitario: una aproximación desde sus actores principales. RIDE, 12(23). DOI: https://doi.org/10.23913/ride.v12i23.983.

Fidalgo-Blanco, Á. (2020). El papel de la innovación educativa durante la pandemia covid-19. Zenodo, 13. DOI: https://doi.org/0.5281/zenodo.4319648.

Hernández, S. R., Fernández, C. C. y Baptista, L. P. (2014). Metodología de la investigación. Mc Graw Hill.

Jiménez, G. J. (2017). Innovación educativa y docencia ¿falla el protagonista? el caso ESCOM. RIDE, 8(15). DOI: http://dx.doi.org/10.23913/ride.v8i15.317.

Leal-Fonseca, D. E., Rojas, L. I., Ortiz-Pradilla, T. y Monroy-Osorio, J. C. (2020). Percepción de los docentes sobre sus acciones innovadoras. Educación y Educadores, 23(3), 427-443. DOI: https://doi.org/10.5294/edu.2020.23.3.4.

Medina, R. A., Ruíz, C. A. y Pérez, N. E. (2019). Diagnóstico de un programa de formación docentes en competencias para el primer año de universidad. Aula Abierta, 48(2), 239250. DOI: https://doi.org/10.17811/rifie.48.2.2019.239-250.

Moriano, J. A., Topa, G., Valero, E. y Lévy, J. P. (2009). Identificación organizacional y conducta intraemprendedora. Anales de Psicología, 25(2), 277-287. Recuperado de: https:// revistas.um.es/analesps/article/view/87631/84411.

Oseda, G. G., Mendivel, G. R. y Durán, C. A. (2020). Potencial de innovación y gestión institucional en la Universidad Nacional de Cañete-Perú. Sophia, (28), 207-236. DOI: https://doi.org/10.17163/soph.n28.2020.08.

Palomera, R., Briones, E. y Gómez, L. A. (2019). Formación en valores y competencias socioemocionales para docentes tras una década de innovación. Praxis \& Saber, 10(24), 93-117. DOI: https://doi.org/10.19053/22160159.v10.n25.2019.9116.

Pascual, A. C., López, P. V. y Hamodi, G. C. (2019). Proyecto de innovación docente: la evaluación formativa y compartida en educación. Resultados de trnsferencia del conocimiento entre universidad y escuela. Revista Iberoamericana de Evaluación Educativa, 12(1), 29-45. DOI: https://doi.org/10.15366/riee2019.12.1.002.

Pesantéz, A. L. (2020). Los grupos de innovación educativa GIE: Una alternativa válida para los docentes universitarios. Instituto Internacional de Investigación y Desarrollo Tecnológico Educativo, 5(15), 10-22. DOI: https://doi.org/10.29394/Scientific.issn.25422987.2020.5.15.0.10-22.

Prendes, E. M. y Cerdán, C. F. (2021). Tecnologías avanzadas para afrontar el reto de la innovación educativa. Revista Iberoamericana de Educación a Distancia, 24(1), 33-46. DOI: https://doi.org/10.5944/ried.24.1.28415.

Ramírez, G. M. y Montoya, J. (2018). La buena docencia y su evaluación desde el punto de vista de las disciplinas en la universidad. REDU, 16(1), 69-85. DOI: DOI: https://doi. org/10.4995/redu.2018.6073.

Ramírez, M. S. y Lugo-Ocando, J. (2020). Revisión sistemática de métodos mixtos en el marco de la innovación educativa. Revista científica de educomunicación, (65), 9-20. DOI: https://doi.org/10.3916/C65-2020-01. 
Ríos, C. P. y Ruíz, B. C. (2020). La innovación educativa en América Latina: lineamientos para la formulación de políticas públicas. Revista Innovaciones Educativas, 22(32), 199212. DOI: https://doi.org/10.22458/ie.v22i32.2828.

Sancho, G. J. (2018). Innovación y enseñanza. De la moda de innovar a la transformación de la práctica docente. Educacao, 41(1), 12-20. DOI: http://dx.doi.org/10.15448/19812582.2018.1.29523.

Sein-Echaluce, M. L., Fidalgo-Blanco, Á. y García-Peñalvo, F. J. (2019). Diseño de un proyecto de innovación educativa docente a partir de indicadores transferibles entre distintos contextos. En M. L. Sein-Echaluce, L., Fidalgo-Blanco, Á y García-Peñalvo, F. J. (Eds.), Actas del V Congreso Internacional sobre Aprendizaje, Innovación y Competitividad. Servicio de Publicaciones Universidad de Zaragoza. DOI:10.26754/CINAIC.2019.0126.

Silva, G. H. (2017). La innovación y educación: variables claves para la competitividad de las empresas. Revista Clío América, 11(21), 20. DOI. http://dx.doi. org/10.21676/23897848.2079.

Zavala-Guirado, M. A., González-Castro, I., y Vázquez-García, M. A. (2020). Modelo de innovacion educativa según las experiencias de docentes y estudiantes universitarios. RIDE, 10(20). DOI: https://doi.org/10.23913/ride.v10i20.590.

Zulueta, C. M. (2020). El papel de la inspección de educación en los procesos de innovación educativa. Estudios socioeducativos, 14(8), 192-205. DOI:http://dx.doi.org/10.25267. 